Goldschmidt 2021 Abstract

https://doi.org/10.7185/gold2021.5335

\section{EPMA analyses of olivine near the boundary with a basaltic glass - the secondary fluorescence effect quantification.}

\author{
DR. MAXIM GAVRILENKO, PHD ${ }^{1,2}$, VALENTINA G. \\ BATANOVA $^{1,2}$, STEPAN KRASHENINNIKOV ${ }^{2}$ AND \\ ALEXANDER V. SOBOLEV ${ }^{1,3}$
}

${ }^{1}$ Université Grenoble Alpes

${ }^{2}$ Vernadsky Institute of Geochemistry and Analytical Chemistry RAS

${ }^{3}$ Institute of Earth Sciences (ISTerre), University Grenoble Alpes Presenting Author: maxim.gavrilenko@univ-grenoble-alpes.fr

Trace element analysis in olivine is challenging but vital, since trace element concentrations in olivine could potentially record conditions of olivine crystallization and, correspondingly, conditions of magma generation, evolution, and/or transport dynamics. Currently, electron microprobe analyses (EPMA) for olivine remain the most popular for many reasons including but not limited to high spatial resolution and high precision. Specially designed EPMA protocols with high beam current and/or long counting time provide low detection limits. However, the effect of secondary fluorescence (SF) should be considered for analysis of certain trace elements in olivine near the olivine-glass boundaries to avoid overestimating the content of elements [1]. The global database of synthetic olivine compositions contains thousands of olivine/glass measurements used for olivine/melt partitioning studies. However, the accuracy of the olivine EPMA data is dependent on quantifying the SFeffect from surrounding glass. Here we report the direct measurement of the SF-effect from basaltic glass on trace elements in olivine obtained in specially designed experiments.

In our experimental study the olivines were homogeneous in each experiment, but ranged in forsterite content $\left(\mathrm{Fo}_{77.3}-\mathrm{Fo}_{90.7}\right)$. Olivines with known trace element compositions, were embedded in basalt powder, and heated to $1250^{\circ} \mathrm{C}$ in 1 -atm furnace for a few minutes and quenched. Because of the short heating duration, negligible reaction occurs between the olivine and melt. As a result, we produced olivine pieces of different sizes embedded in glass, both with known composition (Fig.1). The results of EPMA analyses of olivine before and after experiments were used to quantify the SF-effect in olivine. The data show that the SF-effect, which comes from basaltic glass adjacent to olivine, is most significant for concentrations of titanium and calcium. The main factor is the distance from the interface. Although the most significant SF-effect is observed next to the melt boundary, the SF-effect was detected even in the centers (Fig.2) of large olivine grains (up to $100 \mu \mathrm{m}$ in diameter). Considering that experimental olivines are usually smaller than $50 \mu \mathrm{m}$, we would suggest that global experimental database should be used with caution in terms of olivine trace element contents.

[1] Llovet et al. (2012) J.Phys.D:Appl.Phys.45
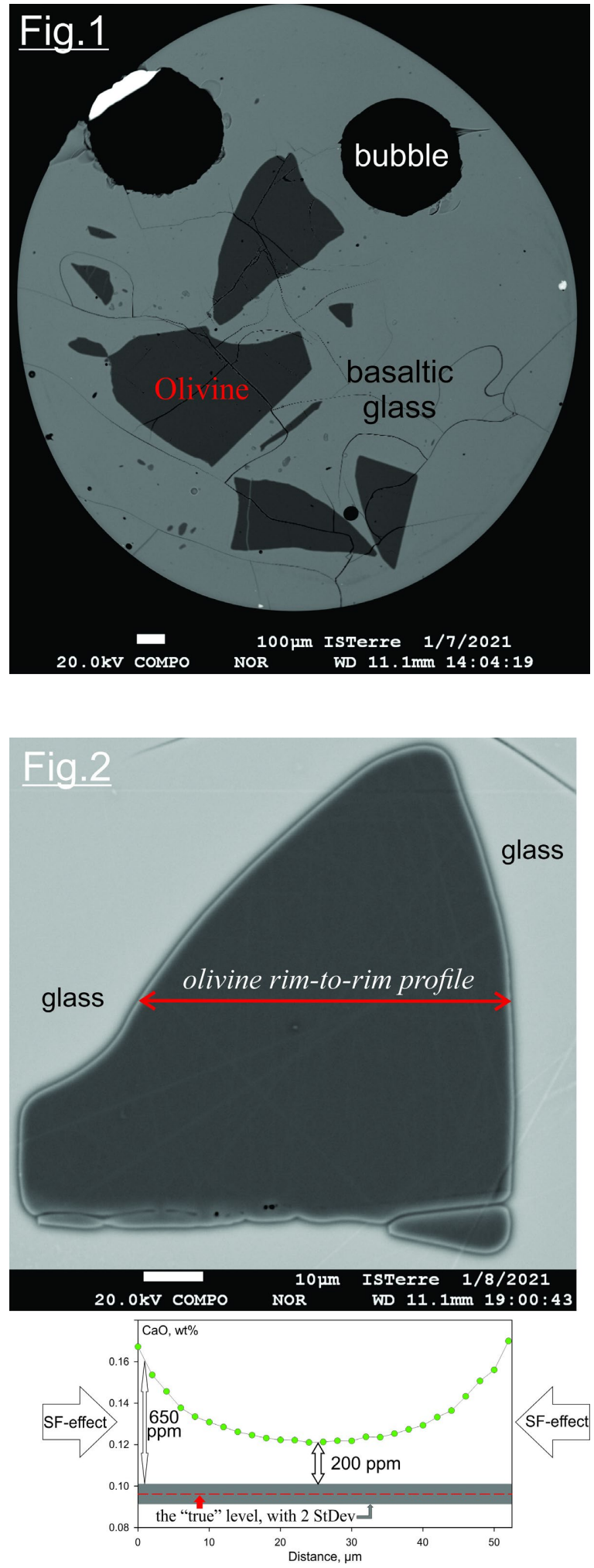